Revista Española de Nutrición Humana y Dietética

www.elsevier.es/dietetica

ORIGINAL

\title{
Cumplimiento de la dieta mediterránea y nivel de actividad física de los usuarios de la web PAFES (Plan de Actividad Física, Deporte y Salud)
}

\author{
Darío López a,*, Alba Pardo ${ }^{\mathrm{b}, \mathrm{c}}$, Gemma Salvador a,c , Angelina González ${ }^{\mathrm{a}, \mathrm{c}}$, Blanca Román ${ }^{\mathrm{c}, \mathrm{d}}$, \\ Juanjo García ${ }^{c, e}$, Mariona Violán ${ }^{c, e}$ y Carmen Cabezas ${ }^{a, c}$
}

\author{
a Departament de Salut, Generalitat de Catalunya, Barcelona, España \\ ${ }^{b}$ Consell Català de l'Esport (CCE), Barcelona, España \\ 'Grupo PAFES, Barcelona, España \\ ${ }^{\mathrm{G} G r u p}$ de Recerca en Nutrició Comunitària (GRENC), Parc Científic de Barcelona (PCB), Barcelona, España \\ e Secretaria General de l'Esport, Programa PAFES, Generalitat de Catalunya, Barcelona, España
}

Recibido el 5 de marzo de 2012; aceptado el 30 de octubre de 2012

\author{
PALABRAS CLAVE \\ Dieta mediterránea; \\ Actividad física; \\ Cumplimiento; \\ Cuestionarios
}

\begin{abstract}
Resumen
Introducción: La web del Plan de Actividad Física Deporte y Salud (www.pafes.cat) permite a los usuarios evaluar el nivel de adhesión a la dieta mediterránea y el nivel de actividad física (AF) mediante dos cuestionarios interactivos: el cuestionario de dieta mediterránea adaptado del estudio "Prevención con dieta mediterránea" (Predimed) y la versión abreviada del Cuestionario Internacional de Actividad Física (IPAQ).

Material y métodos: Se realizó un análisis descriptivo de los resultados obtenidos de ambos cuestionarios durante el periodo 2009-2010.

Resultados: De los 1.376 cuestionarios analizados sobre adhesión a la dieta mediterránea, el $12,5 \%$ de los usuarios tiene un cumplimiento alto; el $67,2 \%$, moderado y el $20,3 \%$, bajo. El 95,8\% utiliza aceite de oliva como principal grasa de adición, el 82,2 y el 78,4\% declaran un consumo bajo de mantequilla y derivados y de bebidas azucaradas respectivamente, y el $70,9 \%$ refiere un consumo adecuado de verduras. Como aspectos negativos, el $82,3 \%$ declara un consumo bajo de legumbres; el 71,6\%, un consumo alto de carne roja, y el 67,6 y el $25 \%$ un consumo bajo de fruta y frutos secos respectivamente. Respecto a la AF, de los 1.221 cuestionarios analizados, el 79,4\% de la población cumple con las recomendaciones de $A F$ saludable, el $36,5 \%$ en un nivel alto y el $42,9 \%$ en un nivel moderado.

Conclusiones: Los cuestionarios vía web pueden ser una herramienta útil en la promoción y la difusión de ambos hábitos saludables. Como propuesta de mejora, se plantea la inclusión de variables sociodemográficas para conocer el perfil de los usuarios que responden a los cuestionarios.

(c) 2012 Asociación Española de Dietistas-Nutricionistas. Publicado por Elsevier España, S.L. Todos los derechos reservados.
\end{abstract}

\footnotetext{
*Autor para correspondencia.

Correo electrónico: dlopezmd@gmail.com (D. López).
} 


\section{KEYWORDS}

Mediterranean diet;

Physical activity;

Adherence;

Questionnaire

\section{Introducción}

El incremento en la disponibilidad de alimentos refinados con elevadas concentraciones de azúcares, grasas y energía (bebidas azucaradas, bollería, entre otros), la desestructuración de las comidas, el bajo nivel de conocimientos y habilidades en la compra y la preparación de alimentos, junto con el elevado nivel tecnológico aplicado a la reducción de la actividad física (AF) en el ámbito laboral, la vida cotidiana y el tiempo libre, predisponen a la población a cierto abandono del patrón tradicional de la dieta mediterránea, así como un aumento del sedentarismo ${ }^{1-3}$. La baja adhesión a la dieta mediterránea y el aumento del sedentarismo están relacionados con un aumento de la prevalencia de las enfermedades crónicas (cardiovasculares, diabetes mellitus [DM] tipo 2, obesidad y ciertos tipos de cáncer), que representan el $60 \%$ de la mortalidad mundial y el $47 \%$ de la morbilidad $^{4}$. La evidencia muestra una asociación positiva entre seguir la dieta mediterránea y disminución de presión arterial $^{5}$, peso corporal, cintura abdominal, concentraciones sanguíneas de colesterol y glucosa e incrementos del colesterol unido a lipoproteínas de alta densidad (cHDL) ${ }^{6}$, así como una mejoría de la función endotelial ${ }^{7}$ y una reducción en los marcadores de inflamación vascular ${ }^{8}$.

Según la Organización Mundial de la Salud (OMS), la inactividad física es la causa principal de un 25\% de los cánceres de mama y de colon, el $27 \%$ de los casos de DM y aproximadamente el $30 \%$ de la carga de cardiopatía isquémica9. Está demostrado que la práctica regular de AF reduce el riesgo de cardiopatía coronaria, accidente cerebrovascular, DM2, cáncer de mama, cáncer de colon y depresión, además de ser un factor clave en el consumo de energía y fundamental para conseguir el equilibrio energético y el adecuado control del peso?.

La dieta mediterránea se caracteriza por un elevado consumo de frutas, verduras, legumbres, frutos secos, cereales (preferentemente integrales), pescados y aceite de oliva como principal fuente de grasa de adición, consumo moderado de productos lácteos (principalmente leches fermentadas y quesos) y bajo consumo de carnes rojas ${ }^{10,11}$. La dieta mediterránea destaca también por la estacionalidad y la sostenibilidad de sus productos y la sociabilidad y la frugalidad de las comidas. En este sentido, la evidencia científica coincide en que seguir la dieta mediterránea es una propuesta de alimentación saludable $e^{6,8,10}$.

Con respecto a la $\mathrm{AF}$, hay consenso sobre la importancia de incrementar el nivel de AF entre la población, y la recomendación se centra en la acumulación de, como mínimo, 30 min de AF moderada (requiere un esfuerzo moderado, que acelera perceptiblemente el ritmo cardiaco y corresponde aproximadamente a 3-6 equivalentes metabólicos [MET]; 1 MET es la cantidad de oxígeno mínima necesaria para las funciones metabólicas del organismo y equivale a $3,5 \mathrm{ml} / \mathrm{kg} / \mathrm{min})^{9,13}$ al menos 5 días a la semana, o $20 \mathrm{~min}$ de $\mathrm{AF}$ vigorosa (requiere gran cantidad de esfuerzo, induce respiración rápida y aumento sustancial de la frecuencia cardiaca y corresponde a > 6 MET $)^{9,13}$ al menos 3 días a la semana o una combinación de ambas ${ }^{12}$, además de proponer un estilo de vida activo (utilización de escaleras, desplazamientos a pie, etc.). 
En 2004, la OMS instó a los países miembros a desarrollar estrategias para la promoción de la salud mediante el binomio alimentación saludable y vida activa, es decir, estableciendo un abordaje conjunto. La OMS recomendaba también trabajar de forma intersectorial, interdisciplinaria y transversal a través de la creación de políticas en distintos ámbitos (educativo, laboral, sanitario, legislativo y comunitario) ${ }^{13}$. En la Unión Europea (UE), se llevó a cabo en 2005 la estrategia "Fomentar una alimentación sana y la actividad física: una dimensión europea para la prevención del exceso de peso, la obesidad y las enfermedades crónicas"14, con el objetivo de promover iniciativas de prevención de la obesidad y otras enfermedades crónicas. Las líneas de la OMS y la UE se concretaron en España en la "Estrategia para la nutrición, actividad física y prevención de la obesidad" (NAOS), que tiene como meta reducir la tendencia ascendente de la prevalencia de obesidad fomentando una alimentación saludable y promoviendo la $A^{15}$. En Cataluña, desde 2006 se impulsa el "Plan integral para la promoción de la salud mediante la actividad física y la alimentación saludable (PAAS)"16, cuyos objetivos son sensibilizar a la población sobre la importancia de una alimentación saludable y la práctica regular de AF. El PAAS se desarrolla mediante acciones informativas y educativas e intervenciones sobre el entorno, principalmente en cuatro ámbitos (educativo, comunitario, sanitario y laboral) y dentro de un planteamiento intersectorial e interdisciplinario. La promoción de la alimentación saludable y la práctica regular de AF son determinantes clave de la salud y el bienestar de las personas y un objetivo prioritario en el marco de las estrategias de salud pública ${ }^{17}$.
Como parte de la estrategia PAAS, se creó en 2007 el Plan de actividad física, deporte y salud (PAFES) ${ }^{18}$, con el objetivo de que los profesionales de atención primaria, con implicación de los ayuntamientos, promuevan la salud prescribiendo AF. En 2009 se creó la web PAFES (www.pafes.cat), dirigida tanto a población general como a profesionales de la salud y de la AF. Por sus características es una plataforma virtual pionera en Cataluña. En el momento del estudio, la web recibía una media de 69 visitas al día.

En la página principal de la web, el usuario encuentra dos cuestionarios interactivos validados: un cuestionario de cumplimiento de la dieta mediterránea (adaptación para la web del cuestionario de dieta mediterránea del estudio Predimed) $)^{6,19}$ y el Cuestionario Internacional de AF (IPAQ, versión abreviada y adaptada para la $w e b)^{20}$, ajustados a un formato divulgativo para facilitar su comprensión y ofrecidos en tres idiomas: castellano, catalán e inglés. Tras responder a los cuestionarios, el usuario obtiene información de su nivel de cumplimiento de la dieta mediterránea y la $\mathrm{AF}$, así como consejos que inciden en aspectos susceptibles de mejora (tablas 1 y 2 ).

El presente estudio tiene como objetivo realizar un análisis descriptivo de los resultados obtenidos en los cuestionarios interactivos de la página web del PAFES durante el periodo 2009-2010.

\section{Material y métodos}

Durante el periodo $2009-2010$ se obtuvo respuesta a 1.450 cuestionarios de dieta mediterránea y 1.236 del nivel de AF.

Tabla 1 Ítems y puntuación del cuestionario de dieta mediterránea

\begin{tabular}{|c|c|c|c|}
\hline Preguntas & Mínimo & Máximo & Respuestas consideradas correctas \\
\hline 1. Aceite de oliva como grasa principal de adición & Sí/no & Sí/no & Sí = 1 punto \\
\hline 2. Consumo diario de aceite de oliva (cucharadas) & 0 & 20 & 2 o más cucharadas $=1$ punto \\
\hline $\begin{array}{l}\text { 3. Consumo diario de verdura y/u hortalizas } \\
(1 \text { ración }=200 \mathrm{~g})\end{array}$ & 0 & 5 & $\begin{array}{l}2 \text { o más (al menos una de ellas en } \\
\text { ensalada o crudas) }=1 \text { punto }\end{array}$ \\
\hline 4. Consumo diario de fruta (piezas) & 0 & 15 & 3 o más al día $=1$ punto \\
\hline $\begin{array}{l}\text { 5. Consumo diario de carne roja, hamburguesas, } \\
\text { salchichas o embutidos ( } 1 \text { ración }=125 \mathrm{~g} \text { ) }\end{array}$ & 0 & 8 & Menos de 1 al día = 1 punto \\
\hline $\begin{array}{l}\text { 6. Consumo diario de mantequilla, margarina o nata } \\
\text { (porción individual }=12 \mathrm{~g} \text { ) }\end{array}$ & 0 & 5 & Menos de 1 al día = 1 punto \\
\hline 7. Consumo diario de bebidas refrescantes/azucaradas & 0 & 10 & Menos de 1 al día = 1 punto \\
\hline 8. Consumo semanal de vino (vasos) & 0 & 14 & 3 o más vasos a la semana $=1$ punto \\
\hline $\begin{array}{l}\text { 9. Consumo semanal de legumbres (1 plato o ración } \\
=150 \mathrm{~g})\end{array}$ & 0 & 10 & 3 o más a la semana $=1$ punto \\
\hline $\begin{array}{l}\text { 10. Consumo semanal de pescado o marisco ( } 1 \text { plato, } \\
\text { pieza o ración: } 125 \mathrm{~g} \text { de pescado o } 4-5 \text { piezas } \\
\text { o } 200 \mathrm{~g} \text { de marisco) }\end{array}$ & 0 & 15 & 3 o más a la semana $=1$ punto \\
\hline 11. Consumo semanal de productos de bollería & 0 & 15 & Menos de 3 a la semana $=1$ punto \\
\hline 12. Consumo semanal de frutos secos (1 ración = $30 \mathrm{~g})$ & 0 & 15 & 1 o más a la semana $=1$ punto \\
\hline $\begin{array}{l}\text { 13. Preferencia de carne blanca sobre la roja } \\
(1 \text { pieza o ración }=125 \mathrm{~g})\end{array}$ & Sí/no & Sí/no & Sí = 1 punto \\
\hline \multirow[t]{2}{*}{ 14. Consumo semanal de sofrito } & 0 & 15 & 2 o más a la semana $=1$ punto \\
\hline & & & Total $=14$ puntos \\
\hline
\end{tabular}


Tabla 2 Valoración del cuestionario de actividad física (AF)

Nivel bajo (no cumple con las recomendaciones)

Nivel moderado (cumple con las recomendaciones en un nivel moderado)

Nivel alto (cumple con las recomendaciones en un nivel alto)
Inactividad física o AF insuficiente para cumplir con las recomendaciones de AF saludable

- $\geq 3$ días de AF vigorosa $\geq 20 \mathrm{~min} /$ día o

- $\geq 5$ días de AF moderada o andar $\geq 30 \mathrm{~min} /$ día o

- $\geq 5$ días de alguna combinación de andar, AF moderada y AF

vigorosa obteniendo $\geq 600 \mathrm{MET}$-min/semana

$\bullet \geq 3$ días de AF vigorosa $\geq 1.500 \mathrm{MET}$ - $\mathrm{min} /$ semana o

- 7 días/semana de alguna combinación de andar, AF moderada y/o vigorosa, acumulando $\geq 3.000 \mathrm{MET}-\mathrm{min} / \mathrm{semana}$

\section{Cuestionario de dieta mediterránea}

Se utilizó y se adaptó un cuestionario de valoración individual del cumplimiento de la dieta mediterránea de 14 ítems, validado del estudio Predimed (http://www.predimed.org). Cada respuesta de cumplimiento otorga un punto, por lo que las puntuaciones posibles van de 0 a 14. Las respuestas se categorizaron según el nivel de cumplimiento de la dieta mediterránea: alto (11-14 puntos), moderado (7-10 puntos) y bajo (0-6 puntos) (tabla 1$)$. Antes del análisis, se realizó una limpieza de datos según una banda de valores aceptables, con lo que se generaron las variables de resultado que se muestran en la tabla 1.

\section{Cuestionario de actividad física}

Se utilizó y se adaptó para la web el cuestionario validado internacionalmente IPAQ ${ }^{20}$. El nivel de AF se categoriza según el protocolo IPAQ basado en las recomendaciones actuales de AF (tabla 2), que valora el nivel de AF en los ámbitos laboral, de ocio, de traslado y doméstico durante los 7 días de la semana previa. El IPAQ registra la intensidad de la actividad según se realice actividad vigorosa o moderada o se camine y también registra información sobre el tiempo de sedentarismo en un día laboral ${ }^{22}$. Los minutos y la intensidad de la AF se transforman en MET, y se asignan los valores de 8,0 MET a la AF vigorosa, 4,0 MET a la moderada y 3,3 MET a caminar. Asimismo se calcula la AF total semanal en MET (MET/min/semana). La variable resultado es el nivel de AF según las recomendaciones: bajo (AF insuficiente para alcanzar las recomendaciones de AF saludable), moderado (cumple con las recomendaciones en un nivel moderado) y alto (cumple con las recomendaciones en un nivel alto) (tabla 2).

La variable tiempo sentado se recodificó en terciles: bajo ( $<5 \mathrm{~h} /$ día), medio (5-8 h/día) y alto ( $\geq 8 \mathrm{~h} /$ día). A su vez, el número de horas diarias de sedentarismo se consideró para definir el nivel de $\mathrm{AF}^{22}$.

\section{Análisis estadístico}

Se realizó un análisis descriptivo. Las variables cuantitativas se expresaron en media \pm desviación estándar, y las ca- tegóricas, mediante frecuencias absolutas y porcentajes. Se utilizó el test de la $\chi^{2}$ para la comparación de las variables categóricas. El nivel de significación estadística se estableció en $\mathrm{p}<0,05$ y el programa estadístico utilizado fue el SPSS, version 15.0 para Windows (Statistical Package for the Social Sciences; Chicago, Illinois).

\section{Resultados}

\section{Dieta mediterránea}

De los 1.450 cuestionarios respondidos, se incluyeron 1.376 en el análisis (el 5,1\% de respuestas inválidas y/o cuestionarios incompletos). El nivel de cumplimiento de la dieta mediterránea (fig. 1) es alto en el $12,5 \%$ de las respuestas, moderado en el $67,2 \%$ y bajo en el $20,3 \%$ (tabla 4 ). El $95,8 \%$ utiliza aceite de oliva como principal grasa de adición, el $82,2 \%$ consume menos de una ración diaria de mantequilla y derivados y el 78,4\%, menos de una bebida azucarada. El

Tabla 3 Cumplimiento de las recomendaciones de la dieta mediterránea $(n=1.376)$

\begin{tabular}{|c|c|c|}
\hline Ítems del cuestionario & Sí, \% & No, $\%$ \\
\hline $\begin{array}{l}\text { Aceite de oliva como principal grasa } \\
\text { de adición }\end{array}$ & 95,8 & 4,2 \\
\hline$\geq 2$ cucharadas de aceite de oliva/día & 84,4 & 15,2 \\
\hline$\geq 2$ raciones de verduras y/u hortalizas/día & 70,9 & 29,1 \\
\hline$\geq 3$ piezas de fruta/día & 32,4 & 77,6 \\
\hline$\leq 1$ ración de carne roja y/o embutidos/día & 28,4 & 71,6 \\
\hline$\leq 1$ ración de mantequilla y derivados/día & 82,2 & 17,8 \\
\hline$\leq 1$ refresco azucarado/día & 78,4 & 21,6 \\
\hline$\geq 3$ copas vino/semana & 23,8 & 76,2 \\
\hline$\geq 3$ raciones de legumbres/semana & 17,7 & 82,3 \\
\hline$\geq 3$ raciones de pescado o marisco/semana & 35,8 & 6,2 \\
\hline$<3$ productos de bollería comercial/semana & 72,5 & 27,5 \\
\hline$\geq 1$ ración de frutos secos/semana & 74,9 & 2,1 \\
\hline Consumo preferente de carne blanca & 68,9 & 31,1 \\
\hline$\geq 2$ raciones de sofrito de verdura/semana & 53,8 & 46,2 \\
\hline
\end{tabular}


Tabla 4 Cumplimiento de las recomendaciones de actividad física $(n=1.221)$

Nivel de actividad física, \%

\begin{tabular}{lll}
\hline Bajo & Moderado & Alto \\
\hline 20,6 & 42,9 & 36,5 \\
\hline
\end{tabular}

$70,9 \%$ consume $\geq 2$ raciones de verduras/día. Por otro lado, el $82,3 \%$ consume menos de 3 raciones de legumbres/semana, el $71,6 \%$ consume $\geq 1$ raciones de carne roja/semana y embutidos y el $77,6 \%$ consume menos de 3 piezas de fruta/ día. Un $25 \%$ de los usuarios declaran un consumo < 1 ración de frutos secos/semana (tabla 3).

\section{Actividad física}

De los 1.236 cuestionarios respondidos, se incluyeron 1.221 en el análisis según el protocolo IPAQ (el 1,21\% de respuestas inválidas y/o cuestionarios incompletos). El 79,4\% de las personas que respondieron el cuestionario cumplen con las recomendaciones de AF saludable, y el 36,5\% está en el nivel alto y el $42,9 \%$, en el nivel moderado. El $20,6 \%$ no cumple con las recomendaciones de AF saludable (tabla 4). La media de tiempo sentado es de 6,1 $\pm 3,0 \mathrm{~h} /$ día, y se observa que los sujetos que pasan sentados más tiempo ( $\geq 5 \mathrm{~h} /$ día) tienden a tener un nivel inferior de AF que los que pasan menos tiempo ( $<5 \mathrm{~h} /$ día), con diferencias estadísticamente significativas $(p<0,05)$.

\section{Discusión}

Los cuestionarios analizados muestran un cumplimiento moderado de la dieta mediterránea y un cumplimiento alto de las recomendaciones de AF saludable.

Respecto al cumplimiento de la dieta mediterránea entre las personas que respondieron al cuestionario interactivo de la web del PAFES, los resultados muestran como aspectos positivos un elevado consumo de aceite de oliva, un bajo consumo de mantequilla y derivados, un bajo consumo de bebidas azucaradas y un elevado consumo de verduras. Sin embargo, el consumo de legumbres de un $82 \%$ de los usuarios es inferior a la recomendación, que se sitúa en $\geq 3$ raciones/semana. El 77,6\% no cumple la recomendación de 3 frutas al día, y tres cuartas partes de los usuarios consumen carnes rojas y/o embutidos $\geq 1$ veces/día (recomendación, $<1 /$ día). Una cuarta parte de los usuarios tienen un consumo $<1$ ración de frutos secos/semana, lo que que se sitúa lejos de la recomendación de la Sociedad Española de Nutrición Comunitaria, que propone un consumo de 3-7 raciones/semana ${ }^{21}$.

Para acercarnos al cumplimento de la dieta mediterránea entre la población española, de los resultados de la Encuesta Nacional de Ingesta Dietética Española (ENIDE) 2011, destaca que el $85,7 \%$ de la población utiliza el aceite de oliva como principal grasa de adición, el $43 \%$ consume diariamente hortalizas y el $35,5 \%$ consume grasa de origen ani-

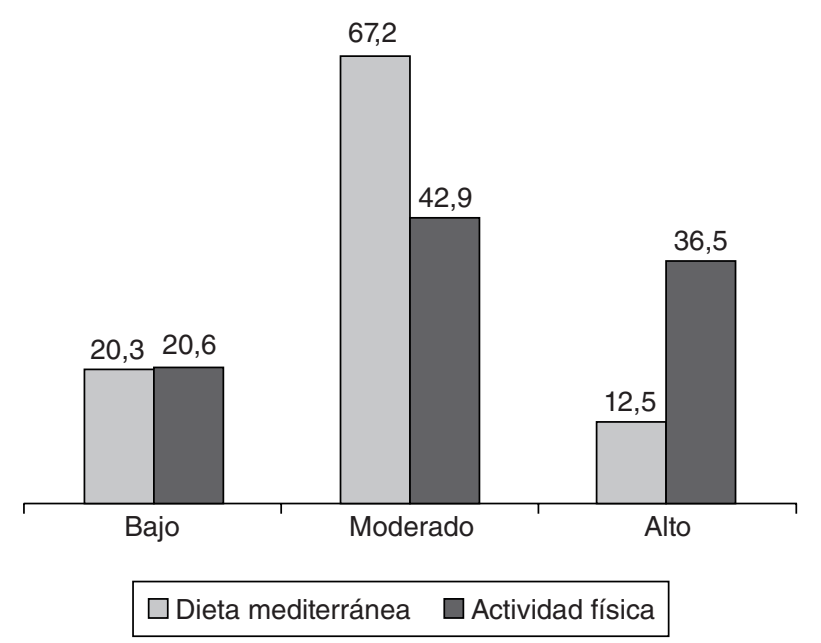

Figura 1 Cumplimiento de las recomendaciones de actividad física y dieta mediterránea.

mal ${ }^{27}$. El cumplimiento de la dieta mediterránea en España ha sido variable en las últimas décadas, y en los últimos años muestra una ligera recuperación. Las comunidades autónomas más cercanas al mar Mediterráneo, entre ellas Cataluña, tienen mayor nivel de cumplimiento que las del interior $^{23}$. En Cataluña, durante el periodo 1992-2003 se observa una mejora del cumplimiento de las recomendaciones respecto al consumo de aceite de oliva, derivados lácteos y frutos secos y menos consumo de carnes rojas y embutidos $^{25}$.

Respecto a los resultados de AF, el presente estudio muestra un bajo porcentaje de personas sedentarias o que no cumplen con las recomendaciones de AF y un alto porcentaje de cumplimiento en comparación con estudios en población catalana (la definición de sedentario utilizada por la Encuesta de Salud de Cataluña 2006 [ESCA 2006] incluye a toda persona que cumpla cualquiera de los siguientes criterios: sentado la mayor parte de la jornada, no realiza ningún tipo de AF o deportiva moderada o vigorosa en el tiempo libre durante la última semana, ha realizado durante un día de la última semana una AF o deportiva moderada $-\geq 20$ min - o vigorosa $-\geq 20$ min - en el tiempo libre y camina $\geq 20$ min menos de 2 días durante la semana) ${ }^{24}$ y española22,26. Estos resultados podrían explicarse por el hecho de que los cuestionarios estén disponibles en una plataforma online, sumado a la importante difusión de la web PAFES realizada entre profesionales del ámbito sanitario, la AF y el deporte, una población muy consciente de los beneficios que comportan una alimentación saludable y una vida activa. Anderson-Bill et $\mathrm{al}^{28}$ observaron que el perfil de usuarios que acceden a los cuestionarios vía web habitualmente corresponde a personas jóvenes, con alto nivel de estudios y buen manejo de internet, mientras que los usuarios que participan en intervenciones de alimentación y AF en internet suelen ser mujeres de mediana edad y con alto nivel de estudios ${ }^{28}$.

Respecto al tiempo que se pasa sentados, considerado un comportamiento sedentario en sí mismo, nuestros resultados indican que las personas que pasan más tiempo senta- 
das tienden a tener niveles más bajos de AF. El tiempo sentado está presente en el tiempo de trabajo y de transporte motorizado y en el tiempo libre. Hay estrategias que muestran que sustituyendo e interrumpiendo el tiempo sedentario por actividades de intensidad leve a moderada, junto con la utilización de transportes y actividades de tiempo libre activos, se incrementa el nivel de actividad física ${ }^{16,18,29}$.

El estudio tiene varias limitaciones; la principal de ellas es la ausencia de variables sociodemográficas que permitan conocer el perfil de los usuarios que respondían a los cuestionarios, lo que además imposibilita la comparación de estos resultados con los de otros de población española. Otras limitaciones importantes son la ausencia de una variable identificativa, que habría permitido identificar las respuestas dobles y/o irreales, y que es un cuestionario disponible en una plataforma virtual, lo que supone un sesgo de selección por el tipo de usuarios.

\section{Conclusiones}

El cumplimiento de la dieta mediterránea es moderado y el nivel de AF es alto entre los usuarios que responden a cuestionarios de la web del PAFES. Según los resultados obtenidos, sería importante incorporar mensajes de promoción del consumo de frutas, legumbres, pescado y frutos secos, así como recomendar la interrupción y, en lo posible, la sustitución de parte de un largo tiempo sedentario por actividades físicas.

No obstante las limitaciones del estudio, los estudios publicados indican que la utilización de cuestionarios interactivos vía web es una forma útil, económica, sostenible y sencilla de obtener información declarada sobre el cumplimiento de la dieta mediterránea y la AF entre la población que responde a este tipo de cuestionarios, que pueden ser un vehículo para la difusión de mensajes específicos para la mejora del cumplimiento de ambas recomendaciones.

Teniendo en cuenta las limitaciones y el alto nivel de respuesta obtenido, se propone incorporar a los cuestionarios variables sociodemográficas (edad, sexo, nivel estudios, situación laboral actual, código postal y/o población) que permitirían, además, adecuar los mensajes de promoción al perfil del usuario. Se podría valorar la posibilidad de ofrecer al usuario consultas online respecto a las recomendaciones que recibe.

\section{Conflicto de intereses}

Los autores declaran no tener ningún conflicto de intereses.

\section{Bibliografía}

1. Jones A, Bentham G, Foster C, Hillsdon M, Panter J. Foresight tackling obesities: future choices-obesogenic environmentsevidence review. Office of Science and Technology. 2007;5:55-61.

2. Varo JJ, Martinez MA, Sanchez A, Martínez JA, De Irala J, Gibney MJ. [Attitudes and practices regarding physical activity: situation in Spain with respect to the rest of Europe]. Aten Primaria. 2003;31:77-84.
3. Sanchez A, Delgado M, Martínez MA, De Irala J. Gender, age, socio-demographic and lifestyle factors associated with major dietary patterns in the Spanish Project SUN (Seguimiento Universidad de Navarra). Eur J Clin Nutr. 2003;57:285-92.

4. World Health Organization. The World Health Report. Reducing risks promoting healthy life. Geneva: World Health Organization; 2002.

5. Perona JS, Cañizares J, Montero E, Sánchez-Domínguez JM, Català A, Ruiz-Gutiérrez V. Virgin olive oil reduces blood pressure in hypertensive elderly subjects. Clin Nutr. 2004; 23:1113-21.

6. Estruch R, Martínez-González MA, Corella D, Salas-Salvadó J, Ruiz-Gutiérrez V, Covas MI, et al. Effects of a Mediterraneanstyle diet on cardiovascular risk factors. Ann Intern Med. 2006;145:1-11.

7. Report on the Global health risks: mortality and burden of disease attributable to selected major risks. Geneva: World Health Organization; 2009.

8. Fuentes F, López-Miranda J, Sánchez E, Sánchez F, Páez J, PazRojas E, et al. Mediterranean and low-fat diets improve endothelial function in hypercholesterolemic men. Ann Intern Med. 2001;134:1115-9.

9. Recomendaciones mundiales sobre actividad física para la salud. Ginebra: Organización Mundial de la Salud; 2010.

10. Esposito K, Marfella R, Ciotola M, Di Palo C, Giugliano F, Giugliano $G$, et al. Effect of a Mediterranean-style diet on endothelial dysfunction and markers of vascular inflammation in the metabolic syndrome: a randomized trial. JAMA. 2004; 292:1440-6.

11. Serra-Majem L, Trichopoulou A, Ngo de la Cruz J et al. Does the definition of the Mediterranean diet need to be updated? Public Health Nutr. 2004;7:927-9.

12. Haskell WL, Lee IM, Pate RR, Powell KE, Blair SN, Franklin BA, et al. Physical activity and public health: updated recommendation for adults from the American College of Sports Medicine and the American Heart Association. Med Sci Sports Exerc. 2007;39:1423-34.

13. Global strategy on diet, physical activity and health. World Health Assembly, 2004 (Resolution WHA 57.17). Geneva: WHO; 2004.

14. Green Book. "Promoting healthy eating and physical activity: a European approach for preventing overweight, obesity and chronic illnesses". Bruselas: European Union Commission European Communities; 2005.

15. Estrategia NAOS para la nutrición, actividad física y la prevención de la obesidad. Madrid: Agencia Española de Seguridad Alimentaria; 2005. Disponible en: http://www.naos. aesan.msps.es/naos/ficheros/investigacion/publicacion1 estrategianaos.pdf

16. Pla Integral per a la Promoció de la Salut mitjançant l'Activitat Física i l'Alimentació Saludable (PAAS). Barcelona: Departament de Salut, Generalitat de Catalunya; 2006. Disponible en: http: //www20.gencat.cat/docs/salut/Home/Ambits\%20tematics/ Linies\%20dactuacio/Salut\%20publica/PAAS/Documents/paas. pdf

17. Pla de salut a Catalunya a l'horitzó 2010: Informe de salut a Catalunya. Disponible en: www.gencat.cat/salut/pladesalut

18. Plan de Actividad Física, Deporte y Salud (PAFES). Barcelona: Generalitat de Catalunya. Disponible en: www.pafes.cat

19. Estruch R, Martínez M, Corella D, Basora J, Ruiz V, Covas M, et al; PREDIMED Study Investigators. Effects of dietary fibre intake on risk factors for cardiovascular disease in subjects at high risk. J Epidemiol Community Health. 2009;63:582-8.

20. Craig CL, Marshall AL, Sjöstrom M, Bauman AE, Booth ML, Aisnworth $\mathrm{BE}$, et al. International physical activity questionnaire: 12-country reliability and validity. Med Sci Sports Exerc. 2003;35:1381-95. 
21. Consejos para una alimentación saludable. Madrid: Sociedad Española Nutrición Comunitaria (SENC), Sociedad Española de Medicina de Familia y Comunitaria (semFYC); 2007.

22. Sjöstrom M, Oja P, Hagströmer M, Smith BJ, Bauman A. Health -enhancing physical activity across European Union countries: the Eurobarometer study. J Public Health. 2006;14:291-300.

23. Bach A, Fuentes C, Ramos D, Carrasco J, Roman B, Bertomeu I, et al. The Mediterranean diet in Spain: adherence trends during the past two decades using the Mediterranean Adequacy Index. Public Health Nutr. 2011;14:622-8.

24. Enquesta i examen de salut de Catalunya 2006. Barcelona: Departament de Salut, Generalitat de Catalunya; 2006.

25. Serra L, Ribas L, Salvador G, Castell C, Román B, Serra J, et al. Avaluació de l'estat nutricional de la població catalana 20022003. Barcelona: Departament de Salut, Generalitat de Catalunya; 2006. Disponible en: http: //www.aesan.mspsi.gob. es/AESAN/docs/docs/evaluacion_riesgos/Cataluna.pdf
26. Bauman A, Bull F, Chey T, Craig C, Ainsworth B, Sallis J, et al; The IPS Group. The International Prevalence Study on Physical Activity: results from 20 countries. Int J Behav Nutr Phys Act. 2009;6:21.

27. Resultados de la primera Encuesta Nacional de Ingesta Dietética Española (ENIDE). Disponible en: http://www.msps.es/ novedades/docs/PresentacionENIDE010311.pdf

28. Anderson-Bill ES, Winett RA, Wojcik JR. Social cognitive determinants of nutrition and physical activity among webhealth users enrolling in an online intervention: the influence of social support, self-efficacy, outcome expectations, and self-regulation. J Med Internet Res. 2011;13:e28.

29. Owen N, Bauman A, Brown W. Too much sitting: a novel and important predictor of chronic disease risk? Br J Sports Med. 2009;43:81-3. 